\title{
The magnetic field and its effects on the solar atmosphere in high resolution
}

\author{
Alan M. Title \\ Lockheed Martin ATC, O/ADBS, B. 252, 3251 Hanover St., Palo Alto, CA, 94304, USA \\ email: title@lmsal.com
}

\begin{abstract}
The Sun's magnetic field is produced throughout the solar interior; it emerges and is dispersed by surface and subsurface flows, and then expands above the surface to dominate the structure of the corona. To resolve the effects of the magnetic field it is necessary to image the interior and measure its rotation and flow systems; track the responses of the magnetic fields to flows in the surface; and to follow the evolution of structures in the corona. Because the Sun is dynamic both high spatial and temporal resolution are essential. Because the Sun's magnetic field effects encompass the entire spherical exterior, the entire surface and outer atmosphere must be mapped. And because the magnetic field is cyclic high-resolution observations must be maintained over multiple cycles.
\end{abstract}

Keywords. Sun, magnetic fields, high-resolution

\section{Introduction}

The last 15 years has been a revolutionary period in Solar Physics because of new observatories on the ground and in space, advances in numerical simulations, and theoretical developments. The new instrumentation and analysis techniques have produced data with a greatly enhanced resolution in time, space, and spectral span. In parallel advances in theory, numerical simulation, and computer technology have allowed significant insights into the complex physical processes that occur in both in the solar atmosphere and throughout the heliosphere. The almost universal commitment of observatories and Principal Investigators to open data policies coupled with fast internet connections has enabled the international community of solar physicists, astrophysicists, plasma physicists, and fluid dynamicists to participate in the analysis process.

The Sun's magnetic field is most probably produced at a range of depths in the solar interior; it emerges and is dispersed by surface and subsurface flows, and then expands above the surface to dominate the structure of the corona and heliosphere. In order to resolve the effects of the magnetic field it is necessary to image the interior and measure its rotation and flow systems, track the responses of the magnetic fields to flows in the surface, and to follow the evolution of structures in the corona. Because the Sun is dynamic, high spatial resolution also implies high temporal resolution. Because of the wide range in temperatures, a broad spectral range is necessary to follow the heating processes above the photosphere. Because the Sun's magnetic field effects encompass the entire spherical exterior, the entire surface and outer atmosphere must be mapped. And because the magnetic field is cyclic, high-resolution observations must be maintained over multiple cycles.

Key to the advances of the last 15 years were the development of active and adaptive optics systems at a number of ground-based observatories and the launch and operation of the YOHKOH, SOHO, TRACE, and RHESSI scientific spacecraft. This year, 2006, has already seen the launch of the Solar X-ray Imager (SXI) on the GOES-N spacecraft 
and will soon see the launches of the STEREO and SOLARB missions. In 2007 new observatories at Big Bear in California and Tenerife in the Canary Islands will start operation. In 2008 the Solar Dynamics Observatory (SDO) will be launched. In the next decade a 4-meter Advanced Technology Solar Telescope and a Frequency Agile Solar Radio telescope should come into operation. These new telescope facilities combined with expected developments in computer technology will lead to an acceleration in our understanding of both interior hydrodynamics and exterior magnetic field dominated processes.

\section{Below the surface}

The science of Helioseismology has allowed mapping of the local and global flows in the solar interior and is just beginning to image the flow systems in and around sunspots. Although Heiloseismology has produced many interesting results, the origin of the Sun's magnetic fields is still a mystery. It is still not established whether the field generation process is limited to the bottom of the convection zone or that fields are generated at multiple depths throughout the convection zone. At present it is difficult to distinguish observationally whether field is pulled to the surface by convection from stored fields in the interior or whether some of the fields are generated by local dynamo processes driven by convection. It is possible, and even probable, that multiple processes are responsible for the surface appearance of new magnetic fields.

The surface distribution of magnetic field is well reproduced by kinematic models that depend on meridional and convective flow systems to distribute the emerged fields over the surface. Helioseismic measurements of the subsurface layers have the potential for providing clues to the dispersal mechanisms and perhaps local and global dynamo processes.

The new high-resolution full disk helioseismograph and vector magnetograph, the $\mathrm{He}$ lioseismic and Magnetic Imager (HMI), on the SDO spacecraft holds promise of imaging the top $10,000 \mathrm{~km}$ of the Sun with sufficient resolution to answer many questions about the origins, emergence, and dispersal of active region magnetic fields. HMI will provide imaging of the magnetic, temperature, and flow structures below spots. In addition it will provide moderate resolution (one arc second) vector magnetograms.

High-resolution observations of sunspots are causing a serious review of the nature of the sunspots and in particular the nature and role of penumbra. It is now clear that sunspots are just part of the plage/moat/sunspot phenomena. The HMI data combined with the high-resolution imagery now possible from the ground (see Fig. 1) should provide the essential data for understanding both the temperature and magnetic structure of sunspots. The continuous coverage of HMI should enable the understanding of the spot emergence and decay processes. The next generation of computers should allow numerical simulation of sunspot phenomena.

\section{The visible surface}

\subsection{Quiet regions}

The measurement of the line of sight magnetic field with Magnetic Doppler Imager (MDI) on $\mathrm{SOHO}$ has provided a nearly unbroken record of full disk magnetograms for a decade. This has allowed the accurate tracking of the evolution of the surface magnetic field. The $\mathrm{SOHO}$ mission was designed to follow individual magnetic features from their origin in active regions and sunspots to their eventual disappearance by merging with magnetic 


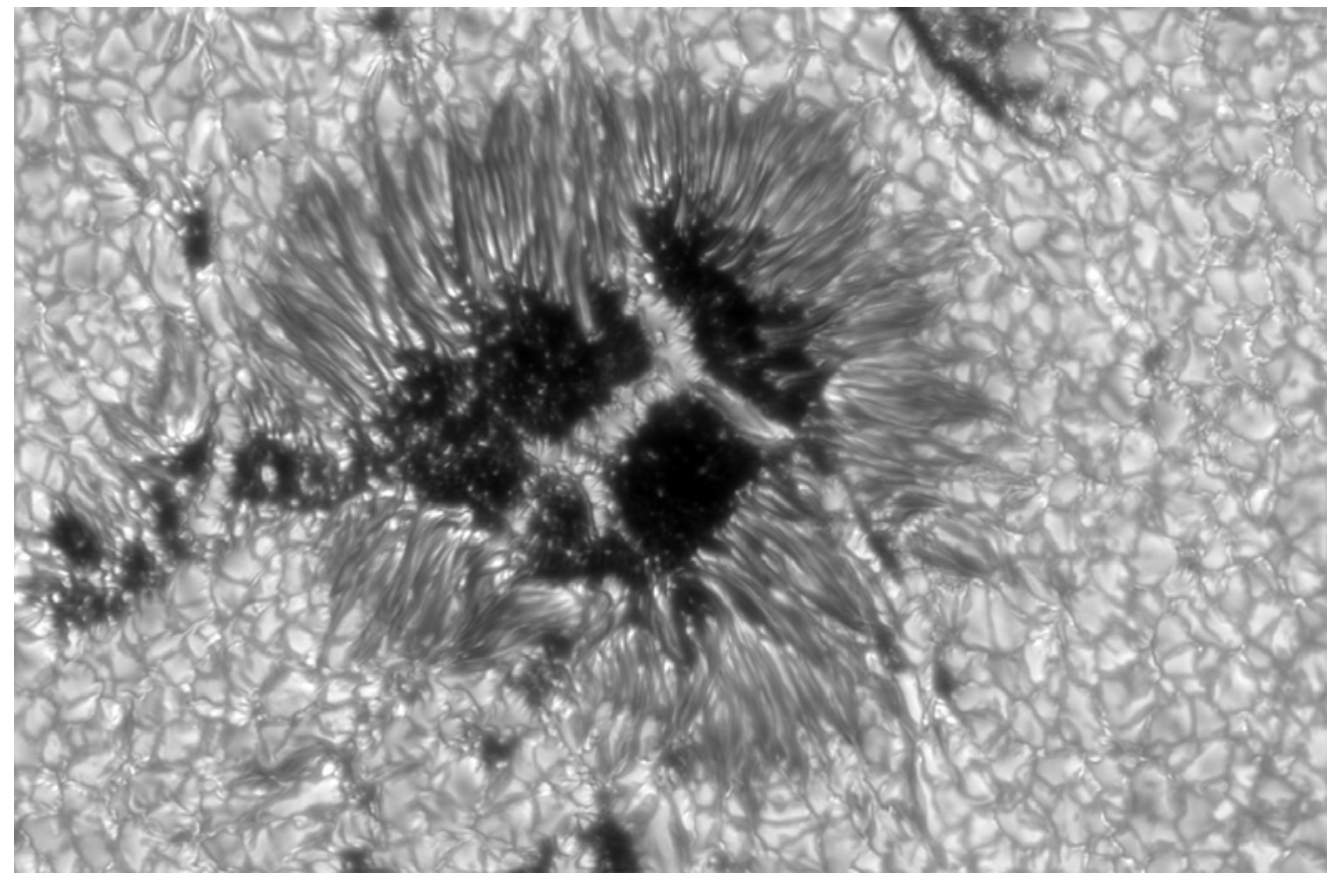

Figure 1. Image of sunspot in G-band taken on 7 June 2006 by T. Berger at the Swedish Solar Telescope, La Palma, Canary Islands, Spain.

flux elements with opposite polarity. This concept was based on the premise that virtually all the magnetic flux important for surface magnetic evolution emerged in active regions. Interestingly, it was discovered that flux emerged everywhere on the surface at a rate such that any magnetic field outside of active regions was replaced in about a day and even flux in active regions lived less than a month.

The MDI data showed that the mixed polarity fields (see Fig. 2) that are seen all over the solar surface, the "Magnetic Carpet", were the result of the constant emergence of small bipoles with a mean flux of $\sim 10^{-18} \mathrm{Mx}$ rather than the result of a surface spreading process acting upon the active region field. The magnetic carpet component of field is not just a kind of magnetic noise, rather it has a number of significant effects. When observed at a resolution of 4 arc seconds the average of the absolute value of the field in quiet Sun was observed to be between 4 and 10 gauss. When observed with the resolution of $3^{\prime}$ the mean flux in quiet Sun usually ranges between \pm 0.1 gauss. As a result the majority of field lines emerging in quiet Sun, even in the network, are connected locally to the sea of magnetic carpet bipoles.

MDI magnetogram data from solar minimum to maximum demonstrated that the amount of flux in the magnetic carpet increases rather than decreases in solar minimum. This suggests that either there is local generation of magnetic fields or that field is stored in the convection zone throughout the cycle and is continuously pulled to the surface by the convective flows. Models of granular convection with rigid lower boundary conditions can create local dynamo action. However, more physically realistic models with open lower boundary conditions do not allow dynamo action because upflow and downflow regions do not mix significantly.

The MDI measurements also have shown that magnetic flux dispersal is not independent of the amount of flux in a local flux concentration. This had been assumed in 


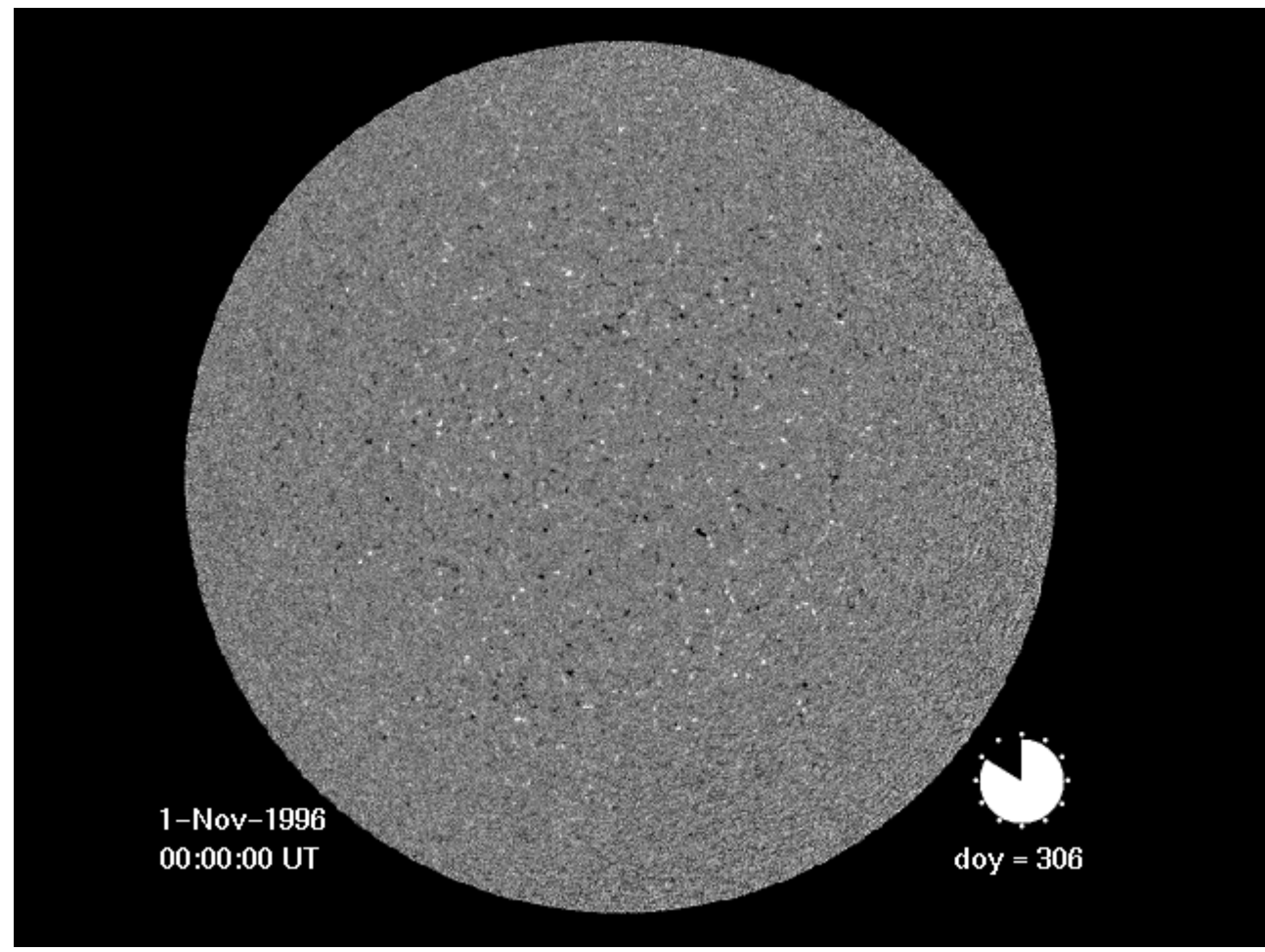

Figure 2. MDI magnetogram at Solar Minimum showing mixed polarity covering the solar diskmagnetic fields. The "Magnetic Carpet", over the entire solar surface.

earlier kinematic flux dispersal models. But larger flux concentrations last longer because it takes more time for the fragmentation and cancellation processes to act. Before this was recognized there was a problem with the kinematic models because they required a diffusion coefficient that was a factor of three larger than measured in order to fit the observed surface flux distributions. The MDI observation that larger concentrations moved more slowly resolved this problem. To measure the diffusion caused by supergranulation, magnetic fields must be tracked over several supergranule turnover times. Only the larger and hence longer-lived magnetic concentrations lasted long enough to measure their displacements. As a result many measurements of the magnetic diffusion coefficient, which results from supergranulation, were under estimates.

Even at MDI deep magnetogram resolution $\left(\sim 4^{\prime \prime}\right)$ the total absolute value of the flux is usually an order of magnitude greater than the net flux averaged over arc minute regions. Observations at higher resolution are suggesting that the total flux may be one or even two orders of magnitude greater than the net flux on arc minute scales. The Stokes Polarimeter of the Solar B Solar Optical Telescope (SOT) in combination with ground based measurements will greatly aid in understanding just how much small scale flux resides on the Sun and how it evolves. The tasks for the new magnetic field measuring instruments on Solar B have been sharpened by the remarkable achievements in adaptive optics and post processing, in particular the fast image capture and adaptive optics operations at the Swedish Vacuum Tower Telescope in La Palma, Canary Islands, Spain and the post processing software developed by the teams at the observatory and the Institute of Theoretical Astrophysics at Oslo University. The ground observations made at the new German, Swedish, and American telescopes, especially chromosphere magnetograms 

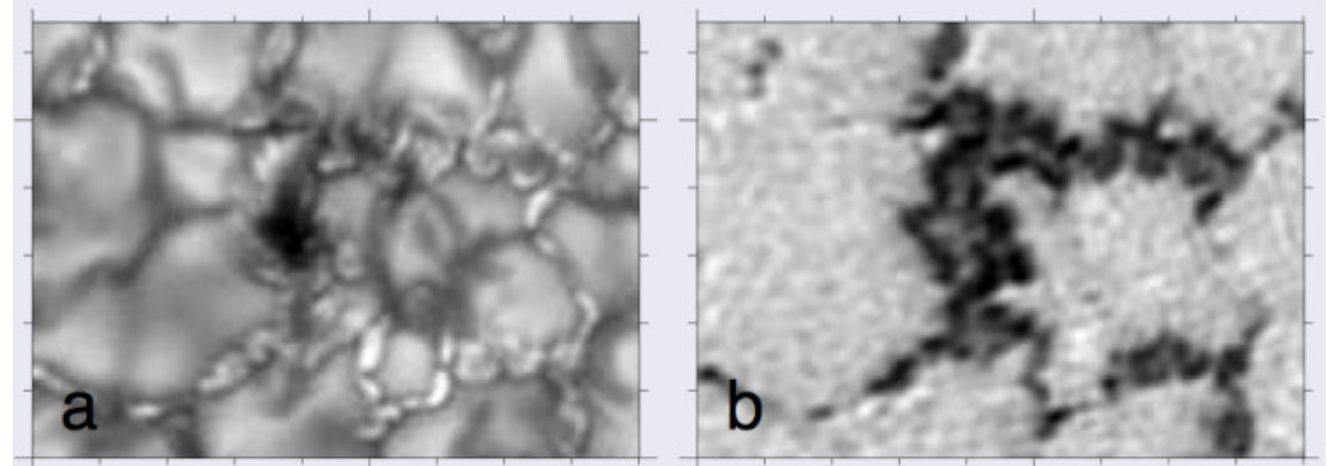

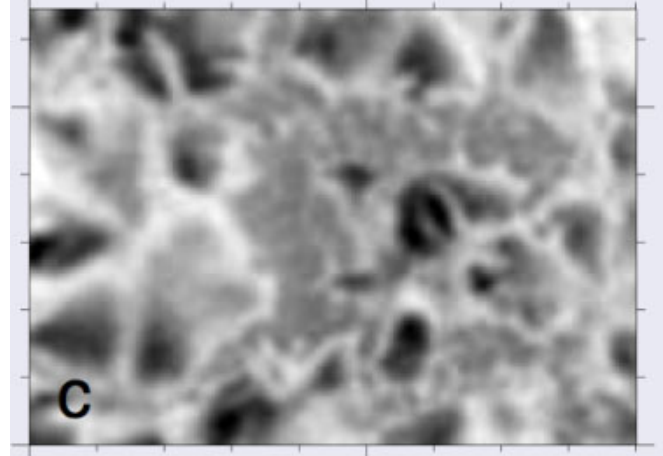

0 arcseconds

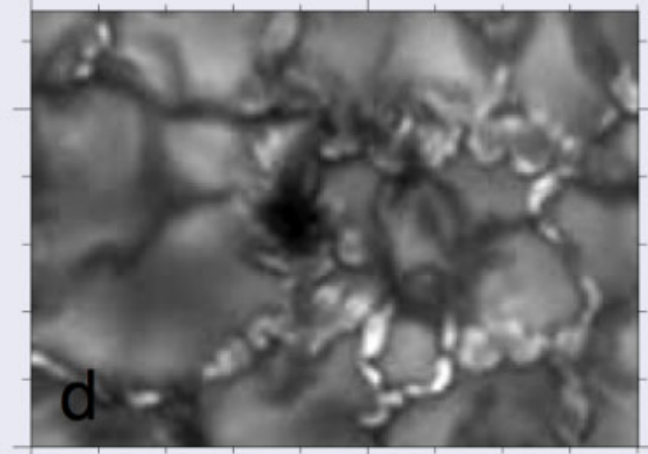

0

arcseconds

Figure 3. Image in white light (a), magnetogram (Fe 6302) (b), dopplergram (Fe6302) (c), and G-band (d). Images from Swedish Vacuum Tower telescope taken by T. Berger.

made in the IR should allow a new understanding of how the flow dominated vector fields in the photosphere evolve into the force free fields in the corona.

Until last few years properties of the smallest magnetic structures have been inferred from observations of the effects of the field on the atmosphere observed in the $\mathrm{CN}$ and $\mathrm{CH}$ bandheads. The presence of the field causes line weakening, which lowers the $\tau=1$ surface. As a result magnetic structures appear as bright features in filter images, $\sim 8 \AA$ wide, centered on the bandheads. All $\mathrm{CN}$ and $\mathrm{CH}$ bright points are contained in the granule boundaries. With adaptive optics and post processing it has been possible to make magnetograms with resolutions comparable to the filter images. These show that the magnetic fields exist in the bandhead bright points and also in the adjacent intergranular lanes (see Fig. 3). Using a combination of narrow band Fabry-Perot filter images, $\sim 0.05 \AA$, and spectral polarimetry in the visible and IR, it now seems that approximately equal amounts of the flux in the intergranular lanes has a strength of greater than a kilogauss and less than 500 gauss.

Magnetogram movies together with spectra and $\mathrm{CH}$ bandhead movies indicate that the bandhead bright points appear when the magnetic field strength exceeds a kilogauss and disappear when the field strength drops below that level. The picture that is emerging is that the granular flows alternately compress and allow the magnetic field to relax toward the equipartion strength of about 300 gauss. As a result the kilogauss regions move about in the intergranular lanes appear, disappear, and merge in a continuous dance modulated by the granular flows. 

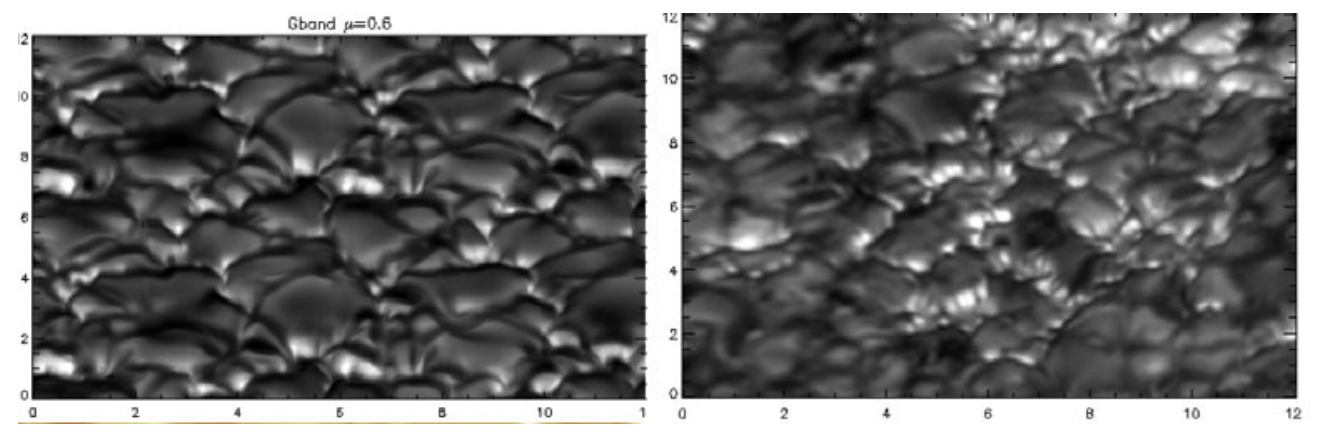

Figure 4. Numerical Simulation of granulation at $\mu=0.6$ (left panel) and observation (right panel). Courtesy Mats Carlsson.

New observations now are showing the nature of the emergence process on very small scales. Numerical simulations indicate that there should be differences between the appearance of small-scale fields created by local dynamo processes and flux loops pushed onto the surface by convective plumes. In the case of dynamo action the magnetic field appears in swirls, "cinnamon buns", whereas convection drives the emergence of small bipoles. The new observations on the ground will provide the best discriminating data because of the larger apertures available. Combining the highest resolution ground observations with precision spectropolarimetry from space will provide critical data for determining the physics of the small magnetic features.

It is well known that the granular pattern lifetime is on the order of ten minutes. This means that the magnetic field concentrations change significantly on that same time scale. This is important because the loops seen in the corona and the structures in the chromosphere and transition region that are attached in to quiet Sun must be evolving on the same time scale. However, this is an upper limit to the time scale because all of the fields are changing position locally. The magnetic structure at any point is changing because of the strength and location of the magnetic fields both locally, in their vicinity, and globally.

A variety of arguments have been made for heating of the corona by twisting, cancellation, merging, and reconnection of small flux tubes. It is now straightforward to create models that can generate enough energy to heat the chromosphere, transition region, and corona, but at present it is not possible to distinguish these different scenarios observationally.

\subsection{Granulation}

Models of surface convection have for more than a decade created simulated images of granulation that are in excellent agreement with the appearance of granulation observed in white light. Recently observations of granulation at the limb with the new one-meter Swedish telescope revealed for the first time the three-dimensional structure of granulation. Initially, the model calculations did not reproduce these observations. This occurred because not enough ray angles were included in the numerical simulations to properly model the granule edges as seen near the limb. This has been corrected and observations and models are in remarkable agreement (see Fig.4). These detailed numerical simulations validate the models made nearly three decades ago that showed that hot walls would occur because the lower density gas in the magnetic flux tubes allowed seeing deeper in to the sides of the granules. As a result the solar luminosity variations caused 
by magnetic fields are much better understood and place the solar luminosity variations seen at the Earth on a firm physical basis.

In regions of dense plage the granulation has been described as "abnormal". Abnormal granulation was characterized by smaller size and lower contrast. This was always somewhat of a mystery because the surface brightness in plage was not different from normal quiet Sun at sensitivity levels of less than $0.1 \%$. The new observations indicate that the granules in plage appear different at lower resolution because magnetic field fills more of the lanes. As a result the lower resolution images show brighter lanes in plage than relatively field-free regions but, in the $100 \mathrm{~km}$ resolution images, contrast between lane and granule center is not significantly different from those in quieter Sun.

\section{The upper atmosphere}

Above the photosphere the temperature of the solar atmosphere initially drops, but about $500 \mathrm{~km}$ above the visible surface the temperature rises from $\sim 4,000 \mathrm{~K}$ at the temperature minimum to $3,000,000 \mathrm{~K}$ in the corona. The processes responsible for the rise are essentially non-thermal and dynamic. Fig. 5 illustrates the complex mixture of cool and hot gas in the chromosphere and corona. In the region of the above temperature minimum the magnetic pressure dominates the gas pressure, while in the photosphere below the gas dynamics control the magnetic field. The problem of heating the chromosphere, transition region, and corona is the problem of understanding how the flow systems in the surface transfer energy to the magnetic fields and then how that energy is dissipated in the atmosphere above.

Observations using the $Y O H K O H$ spacecraft, which imaged the Sun in the soft X-ray region, $\sim 2-3,000,000 \mathrm{~K}$, forced a recognition that magnetic reconnection occurred both often and rapidly in spite of the very high conductivity of the coronal gasses. $\mathrm{SOHO}$ which observes in the EUV $\sim 600,000$ to $2,000,000 \mathrm{~K}$, also showed that magnetic reconnection was a common process. Both $\mathrm{YOHKOH}$ and $\mathrm{SOHO}$ observed the entire Sun, but because of their orbits and the state of technology at the time of their design, they were somewhat limited in both temporal and spatial resolution. The TRACE satellite, which observes in the EUV $\sim 600,000$ to 2,000,000 K, sacrificed full field of view for higher spatial resolution and higher temporal cadence and observes $24 / 7$ for nine months of the year. Together $S O H O$ and TRACE provide complementary observations and normally operate as a single data collection system.

Besides rapid reconnection events, data from the YOHKOH, SOHO, and TRACE missions show that the corona is multi-thermal with no line of sight that can be characterized by a temperature and density (see Fig. 6). A line of sight may and most probably will contain gas in the range of temperatures from 6,000 to $3,000,000 \mathrm{~K}$ even in the absence of flares. Part of the temperature and density mixing may be local. That is, loops may consist of multiple threads that have different temperatures and densities. Some is nonlocal in that there maybe hotter or cooler material both above and below any particular structure under study. Further, loops fluctuate continuously with a time scale of a few minutes and it is difficult to identify an isolated loop for much more than twenty minutes.

A primary goal of the upper atmosphere missions is to collect images and movies in a set of spectral lines that span the temperature range from about $6,000 \mathrm{~K}$ to $3,000,000 \mathrm{~K}$ with a cadence that allows the entire temperature range to be sampled in a time short compared to the rate at which the solar structures are evolving. While the spectral imaging is valuable it suffers from the problem that as the temperature increases or decreases the structure disappears from the image because the line formed in a narrow temperature band. (However, features seldom disappear because of velocity shifts because 


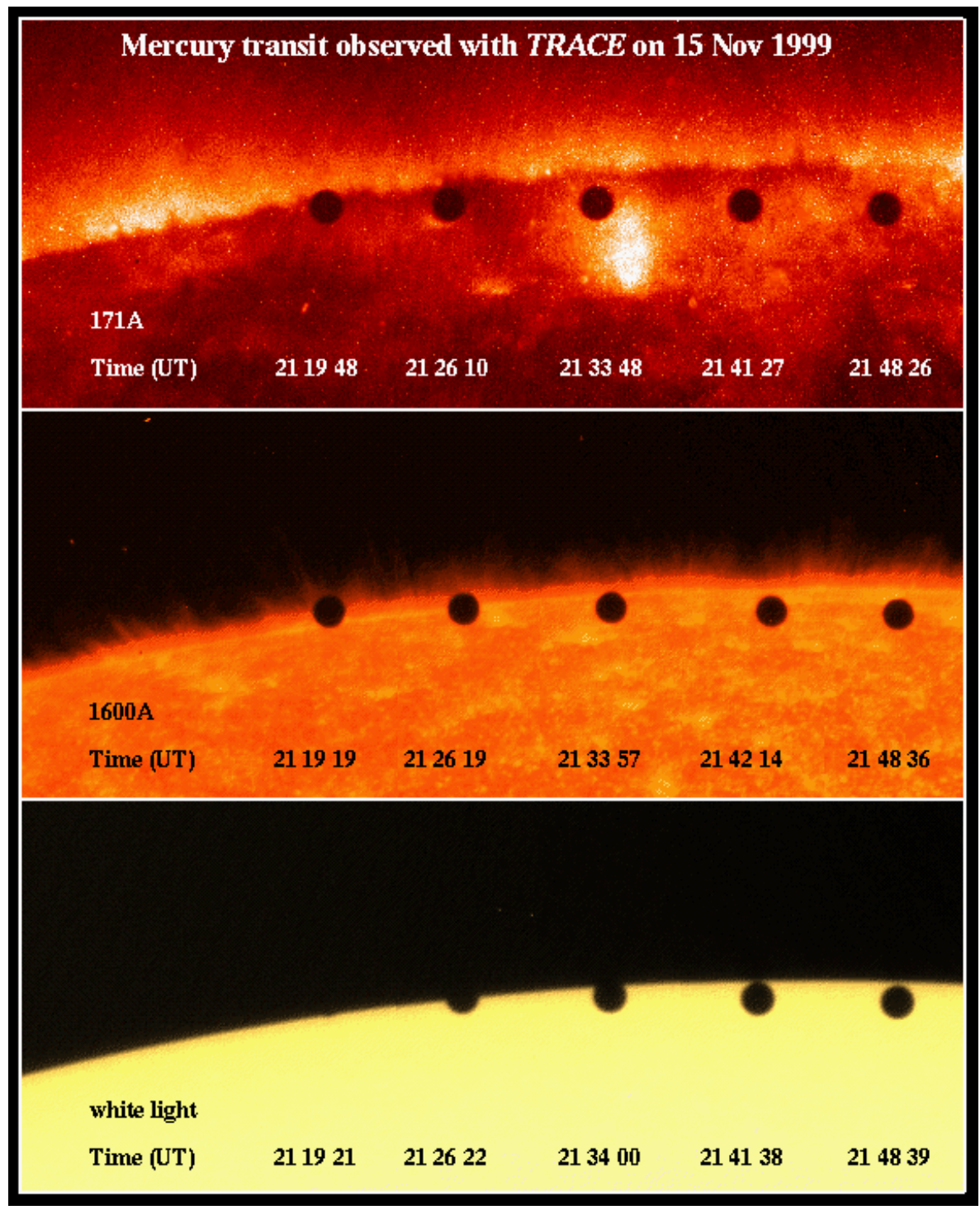

Figure 5. Each panel of this figure is a composite of images of a Mercury transit across the limb of the Sun captured by TRACE. In the vertical direction each set of Mercury images is taken within 30 seconds of time. The upper panel is taken in Fe IX-X $171 \AA$ ( $1 \mathrm{MK})$, the middle in the $1600 \AA$ band which contains C IV $(60,000 \mathrm{~K})$, and the lower image in the continuum $(6,000 \mathrm{~K})$. The diameter of Mercury is $\sim 5 \mathrm{Mm}$. It is clear from the images that the transition region and corona overlap and the transition region extends at least $6 \mathrm{Mm}$ above the visible surface.

the filter bands allow velocities of $\pm 5000 \mathrm{~km} / \mathrm{sec}$.) It is then difficult to establish whether the changes seen in a narrow band are due to temperature or density effects.

To sample the set of four EUV TRACE bands requires about 90 seconds. Most phenomena on the Sun change considerably in 20 to 40 seconds. As a result most TRACE observations have been in a single line or several lines with occasional sample of the full spectral range. Another short coming of both TRACE and EIT (Extreme ultraviolet Imaging Telescope on $\mathrm{SOHO}$ ) is that the 2.5 to 5 million degree corona is not well sampled. The Atmospheric Image Assembly (AIA) on SDO will take full disk observations in 


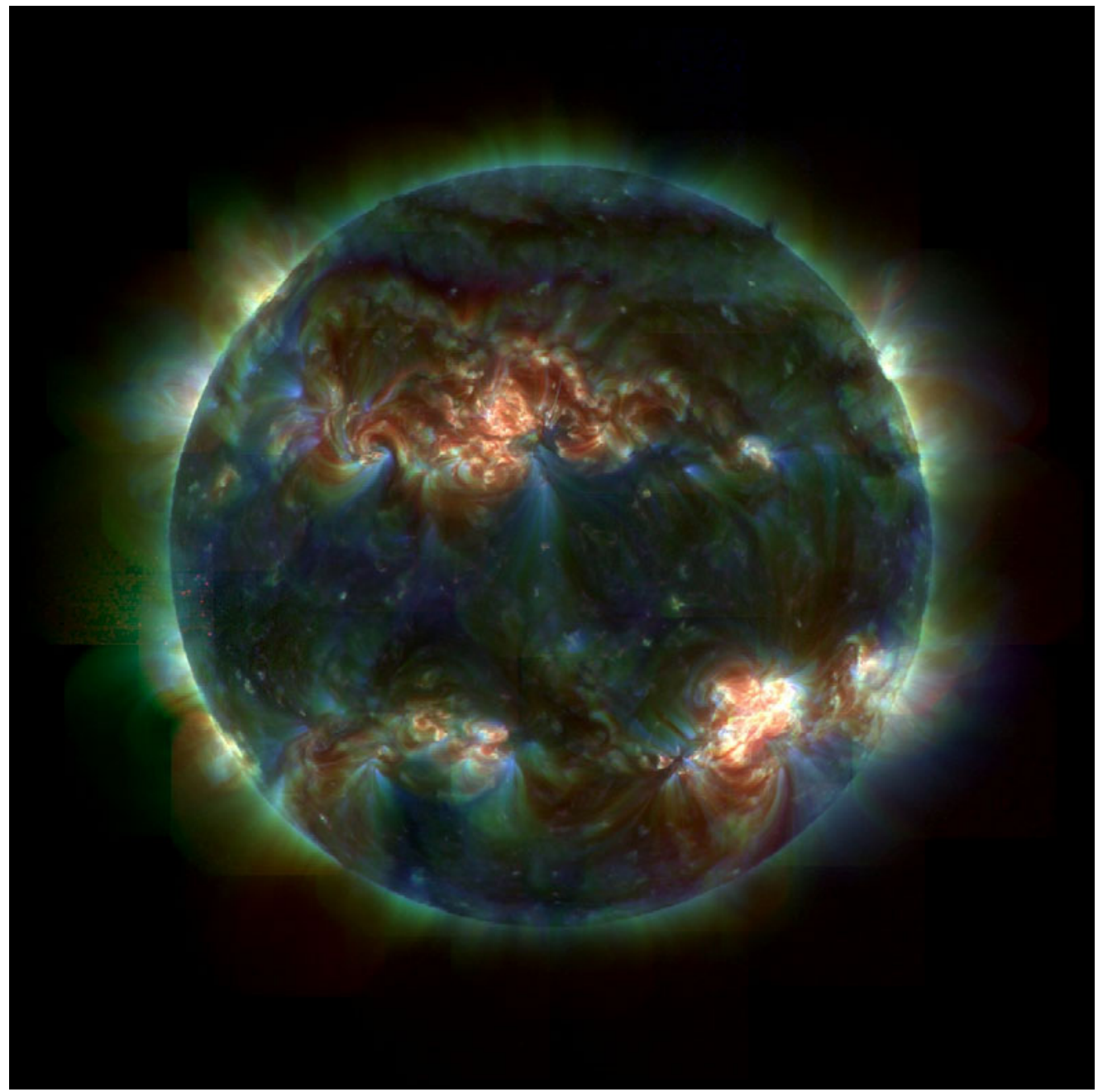

Figure 6. This is a composite of the $171 \AA$ (1 MK-blue), $195 \AA$ (1.5 MK-green), and $284 \AA$ (2 MK-red)) TRACE images. Each TRACE full disk image is a composite of 37 different TRACE pointings. The entire composite consists of 111 separate images and it took $1.5 \mathrm{hr}$ to complete. AIA will take 8 full disk images with nearly the same spatial resolution in $10 \mathrm{sec}$.

8 spectral bands in 10 seconds and with the spatial resolution of TRACE. The SDO data combined with the full disk the X-Ray Telescope (XRT) on Solar B and the new Solar $X$-ray Imager on GOES-N will for the first time acquire images spanning the temperature range from $6,000 \mathrm{~K}$ to $30,000,000 \mathrm{~K}$ over the entire Sun. The 3 -D EUV images from STEREO will contribute greatly to removing some of the ambiguities of the structure and evolution of coronal features. The new Extreme ultraviolet Imaging Spectrograph (EIS) on Solar B will provide extremely valuable data velocity and line profile data, but more is needed.

Because the solar atmosphere is optically thin neither spectral images nor spectra can completely resolve the temperature and density of individual loop structures. The inversion problem is also intrinsically ambiguous. In order to confirm our understanding of loop heating mechanisms forward modeling is required. By using the surface and chromospheric magnetic fields measurements estimates of the magnetic structure in the corona 


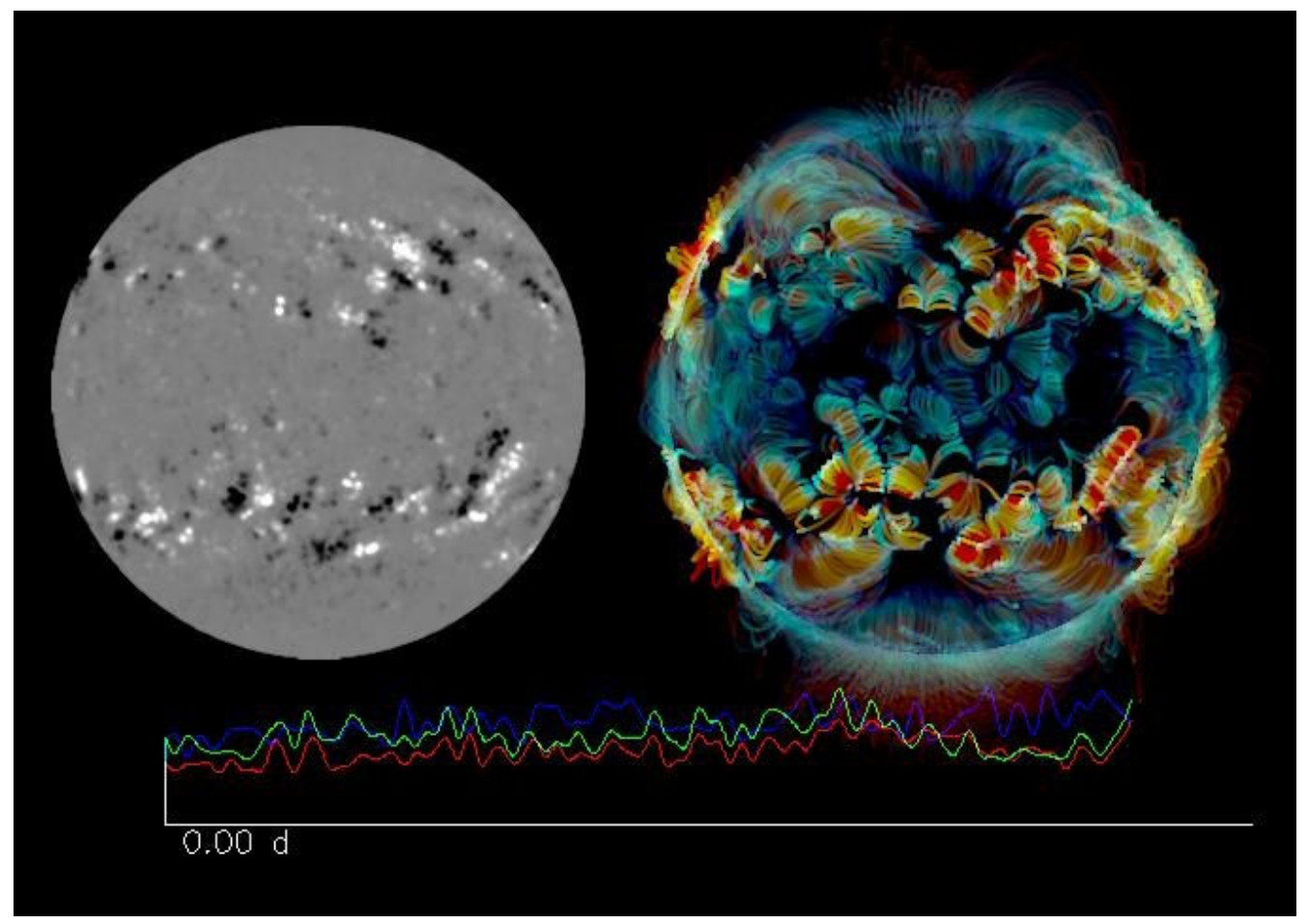

Figure 7. The left hand panel shows a magnetogram and the right hand panel shows the estimated loop structures. The loops are color by temperature $1 \mathrm{MK}$ (blue), $1.5 \mathrm{MK}$ (green), and $>2 \mathrm{MK}$ (red). The plot below show the relative amount of gas in the temperature bands over a 27-day period. The magnetic field lines are a potential field calculation.

can be made. The surface flows can be measured. Then using models of loop heating a model corona can be constructed, which can then be compared with the observations. An example of the synthetic Sun concept is shown in Fig. 7. By continuous refinement of the physics in the models, it should be possible to define the basic physics of at least some of the coronal heating processes. The advances in both computer power and physics based numerical simulations have allowed real progress in understanding some of the major issues in coronal heating.

Another complication to understanding the corona is the lack of knowledge of the transition between the photosphere and the corona. The chromosphere and the transition region are hard to model and are intrinsically dynamic. Observations of the transition region are difficult because properties of materials limit the reflectivity of mirrors in the spectral region that has the most contribution, 50 to $100 \mathrm{~nm}$. But there has been recent work that indicates normal incidence optics can be built to operate efficiently in this spectral region. If this work is successful, then more data on the transition region should become available. Meanwhile, because the coronal observations have demonstrated the importance of these regions significant effort in modeling has been expended and new results are exciting.

The paragraphs above on the corona have emphasized what we do not know. The amazing observations of the past $15 \mathrm{yr}$ have provided many and varied wonderful surprises. We were surprised by how rapid and how pervasive were the magnetic reconnection processes, by how many loops were not in hydrostatic equilibrium, by the observations that most structures less that $\sim 2,000,000 \mathrm{~K}$ were not resolved with one arc second resolution, 


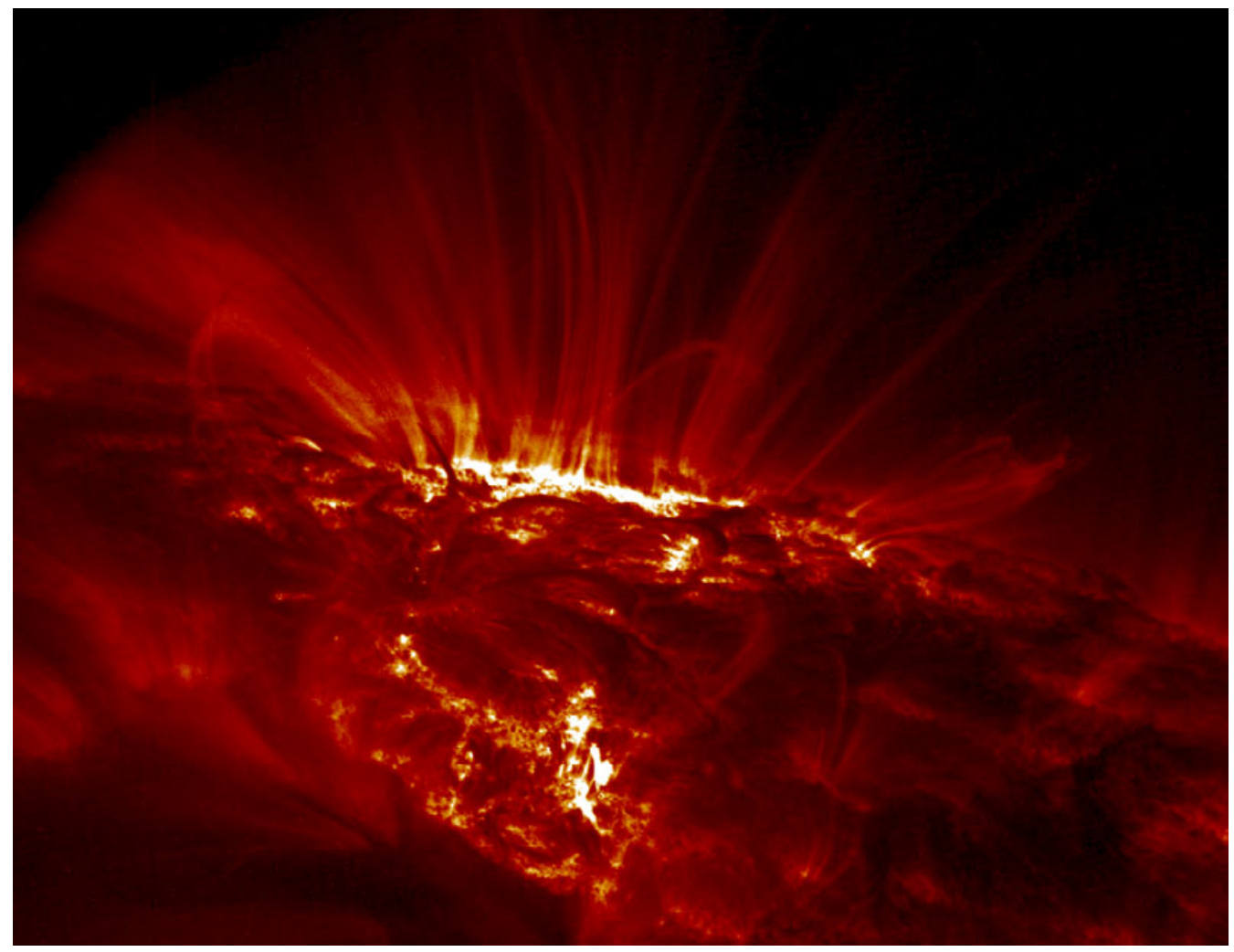

Figure 8. TRACE image in $171 \AA(1 \mathrm{MK})$ showing long loops at the limb that do not increase substantially in cross section with distance from the surface.

and by the observation that the width of most loops did not expand with distance from the solar surface (see Fig. 8). Completely unanticipated were large-scale loop oscillations. Once these oscillations were observed it was surprising that the oscillations damped so rapidly. The discovery of these coronal oscillations has created a new science of "Coronal Seismology".

The topology of the magnetic fields is now recognized an important factor in both the "steady" heating of the corona, the rapid release of energy in solar flares, and the large scale coronal mass ejections. The pervasive emergence of small-scale fields results in continuous reconnection with loops on larger scales. There is a continuous inverse cascade of energy released and transported by the evolution and interactions of smaller and larger magnetic structures. Additionally emergence of new active regions affects adjoining active regions both in the same hemisphere and across the equator. The emergence of flux locally causes rearrangements that can impact the entire Sun.

Surprising observations are the reason science is so exciting. Each new surprise drives new approaches, insights, and understanding. It is not discouraging that many complex interactions are occurring in the Sun; it is exciting, challenging, and important to all of physics and astrophysics. 